\title{
Review of: "Socially responsible investing through the equity funds in the global ownership network"
}

\author{
ADEELA RUSTAM
}

Potential competing interests: The author(s) declared that no potential competing interests exist.

Review comments on "Socially responsible investing through the equity funds in the global ownership network"

Comments by section:

\section{Title of Paper}

The title of the paper is different from the research findings. The title does not represent the study properly. The authors analyzed the connectivity of equity investments to the firms in the global ownership network that are reported as non-compliant with Environment, Social, and Government (ESG) benchmarks, but the title does not cover it. The title should include ESG. To the best of my knowledge, there is a great difference b/w the term ESG and socially responsible investing. The authors should think about it.

\section{Abstract}

Improve the quality of the abstract. The Abstract should follow established schema: 1) reasoning of the research motive; 2 ) review of methods and 3) conclusions.

\section{Introduction}

2. This section presents generally the research gap and the objective of the paper and gives a good view of the concepts, but it does not present the consistency of concepts, gaps, core issues of non-ESG firms, and equity funds the investment firms use as their instruments properly, indirect investing, corporate control which I think it is important and point of concern? The authors should rewrite the introduction section. Please remove the irrelevant sentences and text without references. No stories! Come to the point?

3. On page $3 / 22$ the author mentioned that "Of the issues under the rubrics of Environment, Society, and Governance (ESG), our study focuses on the issues related to environment and military," BUT in the title, the authors are focusing on socially responsible investing..... And in the introduction, the authors are focusing on non-ESG firms. I am literally confused about it. The military and environmental issues do not cover the term ESG? I found many confusing points in the paper.

1. The last paragraph of the introduction section "The remainder of this paper proceeds as follows. ?." It is unnecessary. There is no need to describe sections. The layman can easily understand it. 
2. The research gap is not clear? What is the novelty of conducting this study? Why did you choose? Be specific.

3. Research questions are not clear. However, I don't think you answered all of them. In the discussion / conclusion sections. Think about it.

\section{Literature review}

1. It seems like the purpose of chapters 1 and 2 is the same? (To introduce the reader to the topic and make the rest of the manuscript understandable), improve the readability of the chapters.

2. The cited literature is not enough to support the study findings and theoretical background? Separate chapter 1 and chapter 2 ?

3. To the best of my knowledge, there is a plethora of literature on ESG, non-ESG firm's performance, and equity funds measures. Why did you choose this topic?

a. What is the novelty of your study hypothesis in this regard?

b. What are you contributing to the existing literature? Please explain in detail (theoretical contributions and quantitative contributions).

4. However, its current form lacks a straightforward structure and contains a lot of less relevant material.

5. Remove sentences that do not contain any significant claims, make sure each claim is grounded with the corresponding reference.

6. Your paper lacks theoretical foundations regarding the subject matter? I did not find any theoretical support except for general explanations and empirical literature.

7. The literature review lacks a critical approach? Address the existing literature critically, no need of writing a paragraph about other's findings. Be specific.

8. The authors must cite the most recent research on this topic.

\section{Methodology}

1. What is the actual name of the technique you employed for empirical analysis, I am literally confused. What is the reliability and validity of the study sample size? I don't think so, the study sample is fully representative? Think about it and explain in detail the sample selection and representation?

2. The focus of this study is placed on the secondary data. I wish that the authors give more explanations to illustrate the implication of techniques of analysis. The literature review is insufficient. I strongly suggest that you need to give a comprehensive literature review and the reasons for this choice of technique.

4. Please understand that your methodology needs to be reproducible anybody who reads your instructions should be able to repeat them (step by step) and come to the same results.

\section{Discussion and Conclusion}

1. I have already pointed some reflections for you. What is the main contribution of your paper? I think it is not clearly stated. 
a. What are the policy contributions?

b. According to the results of your research, what you will suggest to globalized capital markets, governments, and policymakers /or what must do to improve?

2. It is also advisable to better justify why you consider your study to be generalizable on an international level.

3. Novelty and significance of your findings are hard to understand, clarify how readers will benefit from your conclusions.

4. The authors used a secondary data set, but the study findings are too narrow. Try to extend all aspects of analyzed results?

5. The policy implications of the research study are not clear? The authors must suggest at least four policy suggestions based on results?

7. Better explain why do you consider your results globally applicable and what are the limitations of your generalizations.

9. Discuss limitations of your methods, provide a synthesis of your results, deeper compare your results with other literature, and propose improvements and directions for future research.

10. Importance of your results needs to be highlighted in the global context. Note that a plethora of literature was published in the field lately therefore, the novelty of your conclusions needs to be groundbreaking. 